\title{
Mutual Coupling Reduction of E-Shaped MIMO Antenna with Matrix of C-Shaped Resonators
}

\author{
Raghad Ghalib Saadallah Alsultan ${ }^{1,2}$ and Gölge Ögücü Yetkin (iD) ${ }^{2}$ \\ ${ }^{1}$ Electronic Department, Technical Institute-Mosul, 00964 Mosul, Iraq \\ ${ }^{2}$ Department of Electrical-Electronics Engineering, University of Gaziantep, 27310 Gaziantep, Turkey \\ Correspondence should be addressed to Gölge Ögücü Yetkin; golge.ogucu@gmail.com
}

Received 31 July 2017; Revised 8 December 2017; Accepted 19 December 2017; Published 18 February 2018

Academic Editor: Yuan Yao

Copyright (C) 2018 Raghad Ghalib Saadallah Alsultan and Gölge Ögücü Yetkin. This is an open access article distributed under the Creative Commons Attribution License, which permits unrestricted use, distribution, and reproduction in any medium, provided the original work is properly cited.

\begin{abstract}
E-shaped multiple-input-multiple-output (MIMO) microstrip antenna systems operating in WLAN and WiMAX bands (between 5 and $7.5 \mathrm{GHz}$ ) are proposed with enhanced isolation features. The systems are comprised of two antennas that are placed parallel and orthogonal to each other, respectively. According to the simulation results, the operating frequency of the MIMO antenna system is $6.3 \mathrm{GHz}$, and mutual coupling is below $-18 \mathrm{~dB}$ in a parallel arrangement, whereas they are $6.4 \mathrm{GHz}$ and $-25 \mathrm{~dB}$, respectively, in the orthogonal arrangement. The $2 \times 3$ matrix of C-shaped resonator (CSR) is proposed and placed between the antenna elements over the substrate, to reduce the mutual coupling and enhance the isolation between the antennas. More than $30 \mathrm{~dB}$ isolation between the array elements is achieved at the resonant frequency for both of the configurations. The essential parameters of the MIMO array such as mutual coupling, surface current distribution, envelop correlation coefficient (ECC), diversity gain (DG), and the total efficiency have been simulated to verify the reliability and the validity of the MIMO system in both parallel and orthogonal configurations. The experimental results are also provided and compared for the mutual coupling with simulated results. An adequate match between the measured and simulated results is achieved.
\end{abstract}

\section{Introduction}

The current wireless communication systems have to fulfill the demands such as high data rates, increased capacity, high quality, and high reliability for different applications. Multiple-input-multiple-output (MIMO) systems provide the suitable technology for these requirements without the necessity of additional bandwidth or transmit power by spreading multiple antennas, with sufficient element spacing, the correct number of elements, and appropriate array geometry or topology [1-3]. In its simplest form, the use of multiple antennas is known as spatial diversity, and the element spacing factor affects the overall performance of the system. In cases where there are limitations of the size and the cost, the spacing between the antennas is insufficient, and this results in mutual coupling, which may influence an impairment of their radiation patterns and changes in the input impedances. Therefore, the isolation between the antennas becomes a crucial task.

Various methods have been studied and analyzed for suppression of mutual coupling and improvement of the isolation between the antennas, including the neutralization technique $[4,5]$, using electromagnetic band gap (EBG) structures [6-8] or etching slots or slits from the ground and forming defected ground structures (DGSs) $[9,10]$. In neutralization technique, an additional coupling is introduced by connecting the two antennas with a thin metal strip. This additional coupling cancels out the coupling between the antennas by properly adjusting the length of the metal strip, and the isolation between the two input ports can be significantly enhanced. In the DGS method, even if the DGS adds an extra degree of freedom in the design, the removal of the metal strip from the PEC or some portions of the substrate require precise micromachining techniques, 


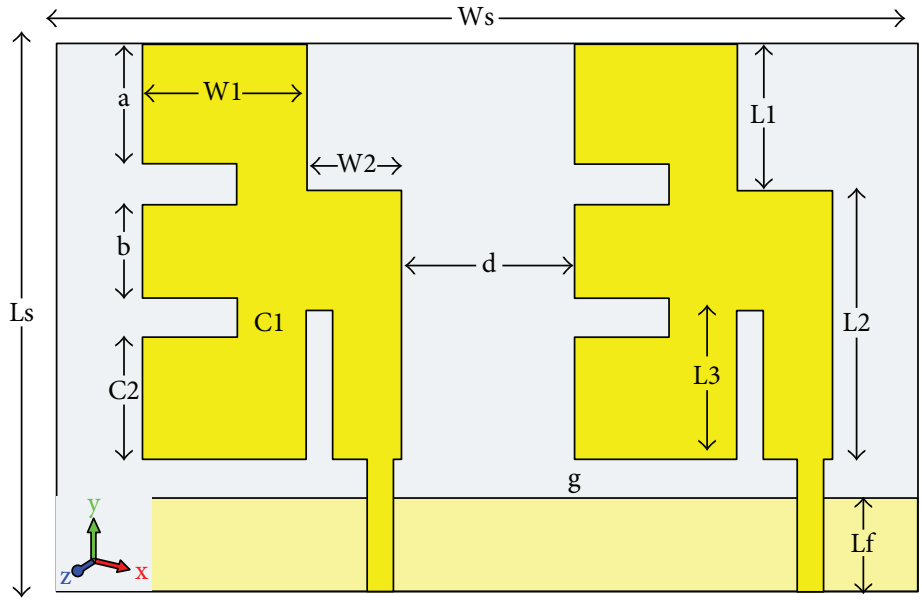

(a)

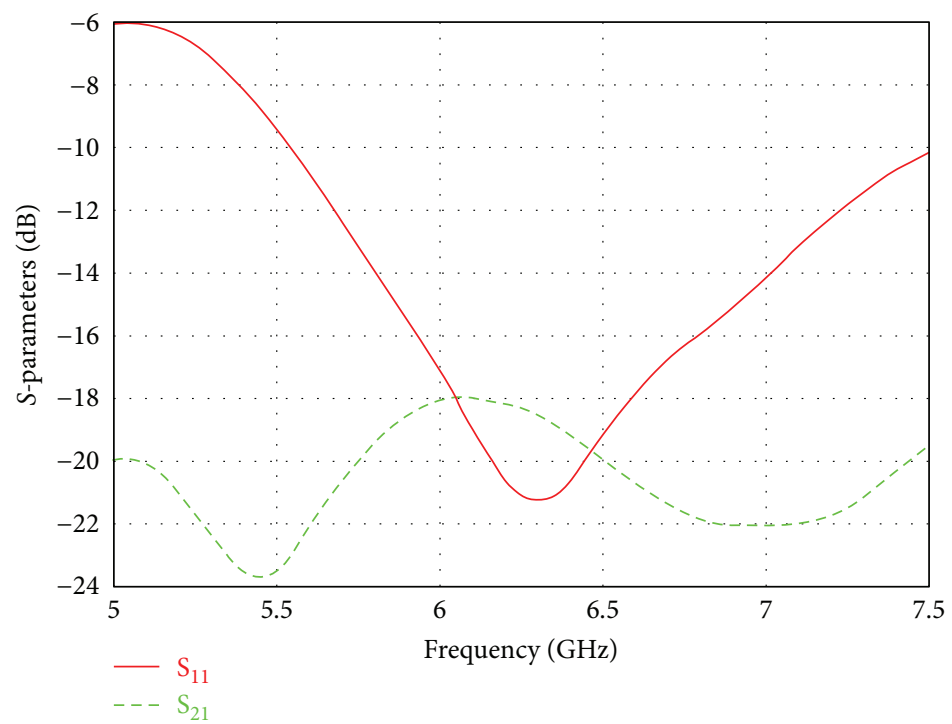

(b)

Figure 1: (a) The proposed MIMO system and (b) S-parameters.

and otherwise, performance degradation can occur. Also, the DGS itself is a slot antenna, which causes an increase in backlobe radiation.

In this work, two E-shaped MIMO antenna systems $41 \times 100 \mathrm{~mm}^{2}$ are presented. The mutual coupling is reduced by placing the EBG metamaterial structure consists of a $2 \times 3$ matrix of C-shaped resonator (CSR) between the antenna elements. The same $2 \times 3$ CSR matrix has been placed and examined between the two antennas, in both cases when the two antennas are in a parallel or orthogonal configuration. The CSR's effective permittivity and permeability are also provided and obtained by the use of Nicholson-RossWeir (NRW) method [11]. It is showing that the proposed structure possesses a wideband metamaterial behaviour.

\section{Antenna Design and Decoupling Structure}

2.1. Antenna Design. Since the goal is to deal only with the mutual coupling mechanism, a simple MIMO system consisting of two E-shaped monopole antennas has been chosen to demonstrate the capability of the approach in this paper. The antennas are placed in parallel and operating between 5 and $7.5 \mathrm{GHz}$ as shown in Figure 1(a). The size of each antenna is chosen such that the system resonates at $6.3 \mathrm{GHz}$, providing an impedance bandwidth of $34.42 \%$ as shown in Figure 1(b). The antennas are placed on FR4 substrate having a dielectric constant $\varepsilon_{r}=4.3$ with a loss tangent of 0.025 and thickness $h=1.6 \mathrm{~mm}$. The separation between the antenna elements is $d=0.42 \lambda_{\mathrm{o}}$. All other dimensions are given in Table 1.

A prototype of this antenna system is shown in Figure 2. It is tested by using an HP8720D network analyzer. Figure 3 shows the comparison between simulated and measured $S$-parameters' results of the proposed MIMO system. It is noticed that there is little difference between the simulation and measurement results. This difference can be considered acceptable as long as its effect on the resonant frequency is not high and the frequency band does not shift much. The discrepancies are mostly due to the insertion loss of SMA connectors, surrounding environment that influences on 
TABLE 1: Dimensions of the parameters of the MIMO antenna system.

\begin{tabular}{lccccccccccccccc}
\hline Parameter & Ws & Ls & W1 & W2 & Wf & L1 & L2 & L3 & Lf & a & b & c & c1 & g & d \\
\hline Dimension $(\mathrm{mm})$ & 100 & 41 & 19 & 11 & 3 & 11 & 20 & 11 & 10 & 9 & 11 & 10 & 2 & 2 & 20 \\
\hline
\end{tabular}

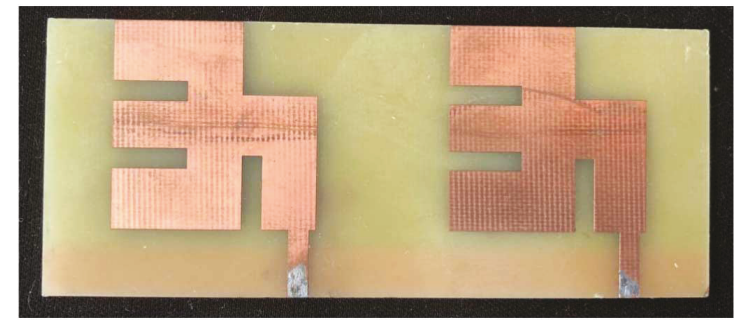

Figure 2: Fabricated antenna.

wave reflection, or the fabrication tolerances. As can also be observed from Figure 3, the coupling between the antennas is about $18 \mathrm{~dB}$ in simulation and $16 \mathrm{~dB}$ in measurements. To reduce this coupling and increase the isolation, the CSRs, whose design procedure will be explained in the next section, are used.

2.2. Analysis of the Unit Cell. Figure 4(a) shows the 3D view of the geometrical configuration of the proposed unit cell of CSR. The CSR unit cell can be modelled by an equivalent circuit using a capacitor and an inductor element. In this case, the gap in CSR can be represented by a capacitor $\mathrm{C}$ while the C-shaped strip can be represented by the inductor $\mathrm{L}$ as shown in Figure 4(b) [12]. $C_{m}$ denotes the coupling to the next element of the array of CSRs. The dimensions of the unit cell are given in Table 2. The model of the structure is first designed and tested on an FR4 substrate with a relative dielectric constant of 4.3 and a thickness of $1.6 \mathrm{~mm}$ as shown in Figure 5(a).

It is obvious that the placement of a sheet of resonators of infinite extension between the two antennas is not possible. Therefore, the designed structure consisting of a $2 \times 3$ matrix of CSR is studied. To investigate the characteristic properties such as the effective permittivity and effective permeability of this matrix, the Nicolson-Ross-Weir (NRW) method is used [11]. The effective permittivity and permeability can be written as follows:

$$
\begin{aligned}
\varepsilon & =\left(\frac{k}{k_{0}}\right)\left(\frac{1-\Gamma}{1+\Gamma}\right), \\
\mu & =\left(\frac{k}{k_{0}}\right)\left(\frac{1+\Gamma}{1-\Gamma}\right),
\end{aligned}
$$

where $k_{0}$ is the propagation constant of free space. Here,

$$
\Gamma=\left[\frac{S_{11}^{2}+S_{21}^{2}+1}{2 S_{11}^{2}}\right] \pm \sqrt{\left[\frac{S_{11}^{2}+S_{21}^{2}+1}{2 S_{11}^{2}}\right]^{2}-1}
$$

The sign is chosen such that $|\Gamma|<1$. The results are provided in Figure 5(b), and it is observed that $2 \times 3$ matrix of CSR structure exhibits negative permittivity and negative permeability in the frequency band of interest around the operation frequency. So this structure can be considered as a double-negative (DNG) medium. This metamaterial property of the $2 \times 3$ matrix of CSR structure has the ability to suppress the surface waves and the space waves (which are considered to be two significant sources to enhance the coupling between the closely antennas), by impeding wave propagation in the area between the MIMO antenna elements.

\section{Measurements and Results}

3.1. Parallel Configuration. In this section, the proposed decoupling technique is utilized to enhance the isolation between MIMO antennas by placing a $2 \times 3$ matrix of the CSR structure as shown in Figure 6(a). The CSR matrix can block the surface waves inside the substrate of the antenna and guide them in another direction by creating an indirect signal with the additional coupling path that opposes the signal going directly from element to element. It is known that if the two waves have comparable amplitudes, then the two waves add up destructively resulting in the suppression of mutual coupling [13]. It is evident from the simulation results in Figure 6(b) that the proposed technique achieves a reduction in mutual coupling and results in an isolation of $30 \mathrm{~dB}$ with an improvement in the isolation about $12 \mathrm{~dB}$ at the resonant frequency.

The prototype of the antenna system is printed as shown in Figure 7. The comparison between the measured $S$-parameters of the proposed MIMO system with and without MTM-CSR matrix structure is shown in Figure 8, and it is observed that there is an improvement in $S_{21}$ with the MTM-CSR matrix structure from $16 \mathrm{~dB}$ to $26 \mathrm{~dB}$. Figure 9 shows the simulated and measured $S$-parameters with the MTM-CSR matrix structure of the proposed MIMO system, where there is a good match between the results.

The influence of decoupling can be observed by visualizing the surface current plots on the antenna array without CSR matrix structure; strong surface current can be observed on the patch antenna on the right-hand side when the patch antenna on the left is excited as shown in Figure 10(a). The surface current is suppressed by the introduction of CSR matrix structure in the middle of antennas as shown in Figure 10(b). The surface current caused by mutual coupling is suppressed by placing the metamaterial decoupling structure. The envelope correlation coefficient (ECC) is a metric that affords essential knowledge about the performance of the array for MIMO systems. The lower the correlation, the higher data throughput can be supported by the MIMO antenna. It is viewed as that antenna with a correlation coefficient under 0.7 has the ability to provide a rich diversity 


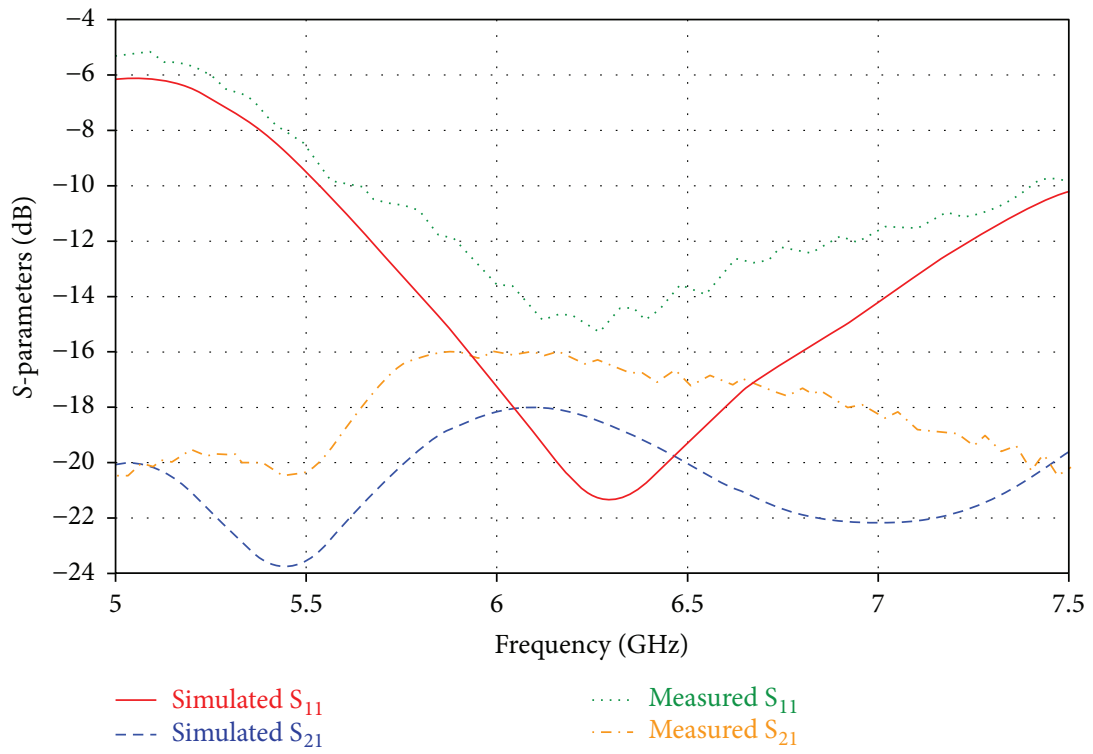

FIgURE 3: Comparison between the simulated and the measured $S$-parameter results.

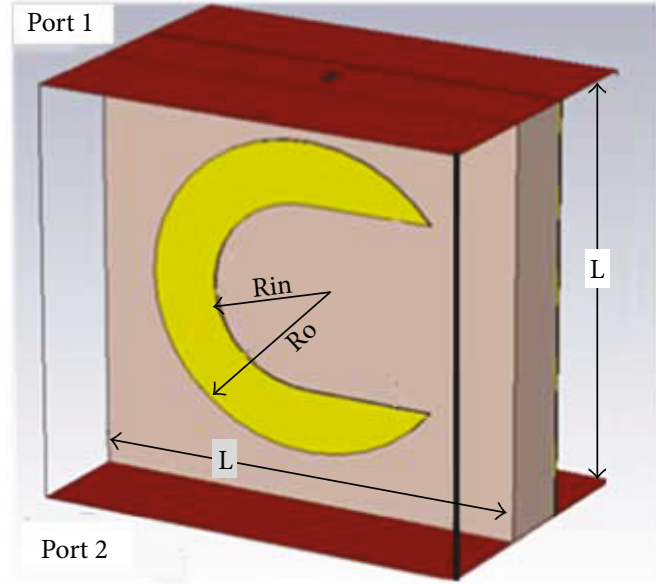

(a)

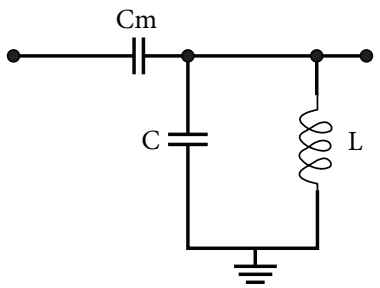

(b)

FIGURE 4: The unit cell of CSR structure. (a) 3D view of the unit cell. (b) The equivalent circuit of the CSR.

performance [14]. The ECC can be calculated from S-parameters as given below [14]:

$$
\rho_{e}=\left|\frac{S_{11}^{*} S_{12}+S_{21}^{*} S_{22}}{\left(\sqrt{1-\left|S_{11}\right|^{2}-\left|S_{21}\right|^{2}}\right)\left(\sqrt{1-\left|S_{22}\right|^{2}-\left|S_{12}\right|^{2}}\right)}\right|^{2} .
$$

TABLE 2: Dimension of unit cell.

\begin{tabular}{lccccc}
\hline Parameter & L & Rin & Ro & g & h \\
\hline Dimension $(\mathrm{mm})$ & 8 & 1.8 & 3 & 3.6 & 1.6 \\
\hline
\end{tabular}

The ECC is calculated for MIMO system, without and with MTM-CSR matrix structure and is plotted in Figure 11. From the plot, it can be noted that the ECC is under 0.0001, which means there is a good isolation between the two antennas in the MIMO system. The diversity gain (DG) is another critical parameter that must be taken into account while evaluating the MIMO performance since it gives an idea about the reliability of the MIMO system. The DG has been calculated using the mathematical expression [14]:

$$
\mathrm{DG}=\sqrt[10]{1-|\rho|^{2}}
$$

The higher the value of diversity, the better the isolation and vice versa. As observed from Figure 12, there is an improvement in the DG with MTM-CSR matrix. The DG of the proposed system with MTM-CSR matrix approaches about $9.99 \mathrm{~dB}$ within the frequency band. Finally, Figure 13 describes the simulated total efficiency of the MIMO array when the antennas are in the parallel configuration in the absence and presence of the decoupling structure MTM-CSR matrix. It is apparent that the total efficiency shows an improvement within the operating frequency band of the antennas in the presence of the proposed decoupling structures.

3.2. Orthogonal Configuration. The performance of a MIMO system can also be improved by using the antennas with the same gain in a given direction, but with orthogonal polarization, which is known as polarization diversity. In order to study this concept, the antenna elements are placed such that 


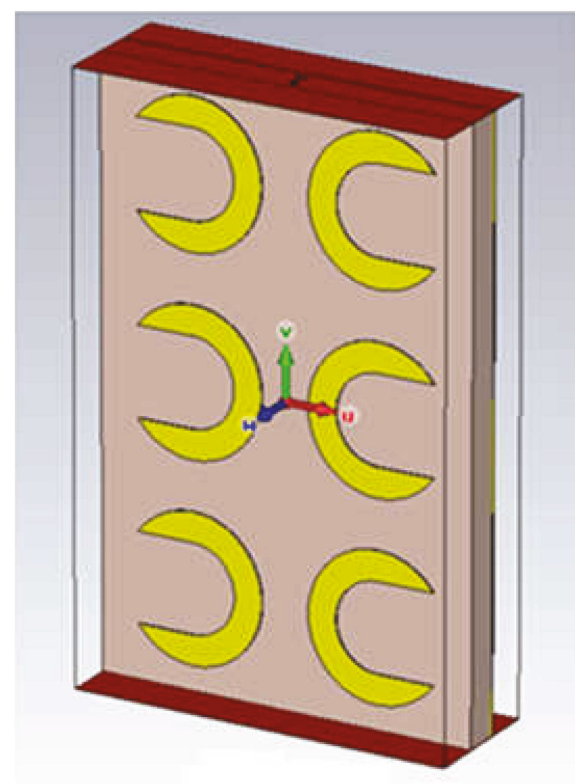

(a)

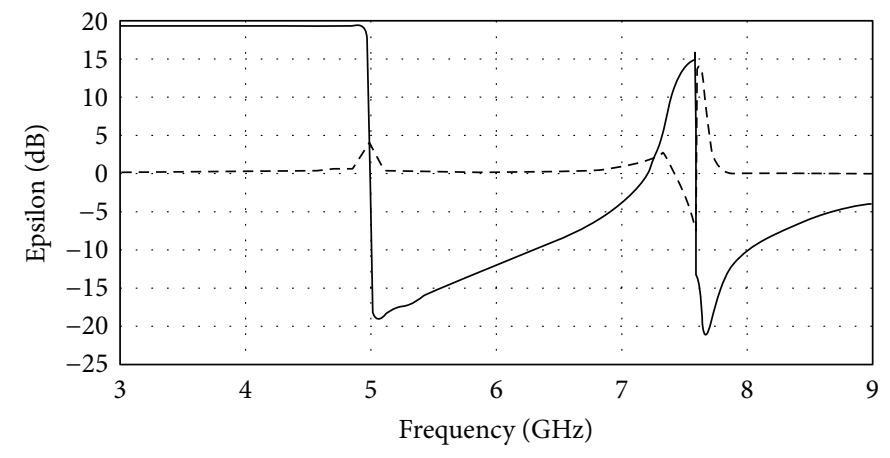

- Eps_re

--- Eps_im

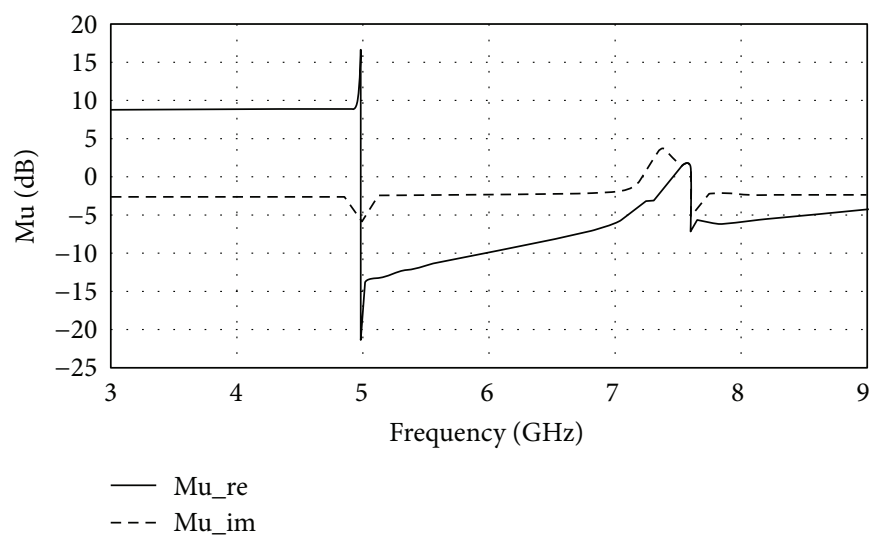

(c)

FIGURE 5: (a) $2 \times 3$ matrix of MTM-CSR, (b) effective permittivity, (c) and effective permeability.

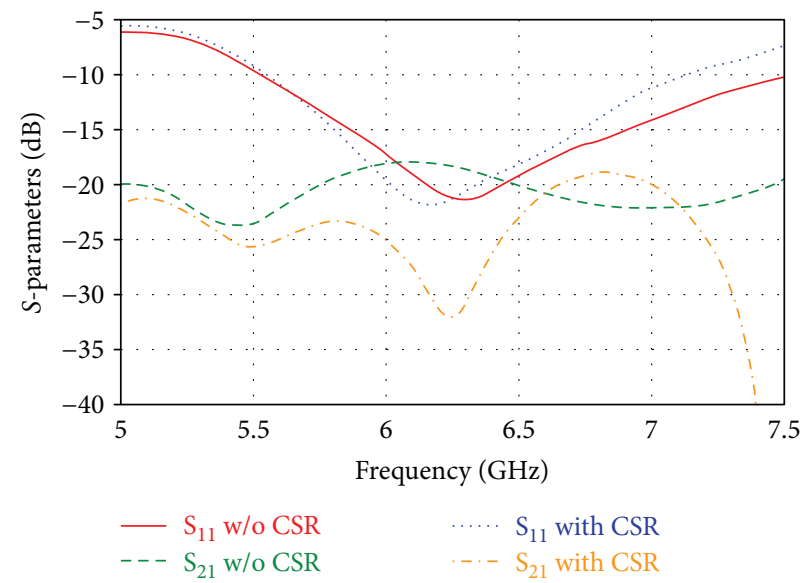

(a)

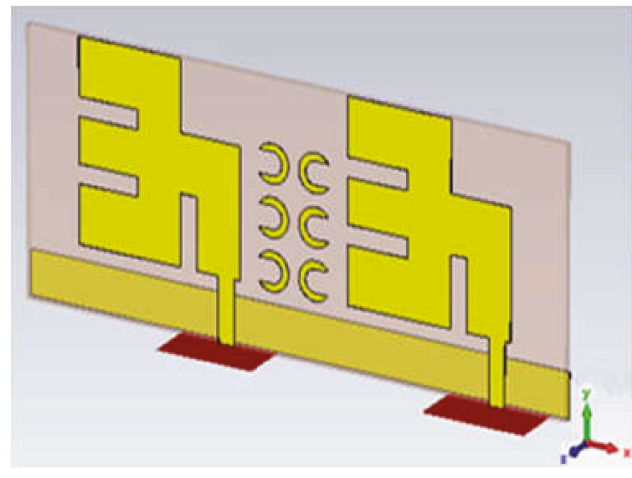

(b)

FIGURE 6: (a) MIMO system antennas with CSR matrix structure. (b) Simulated S-parameters with and without CSR matrix structure. 


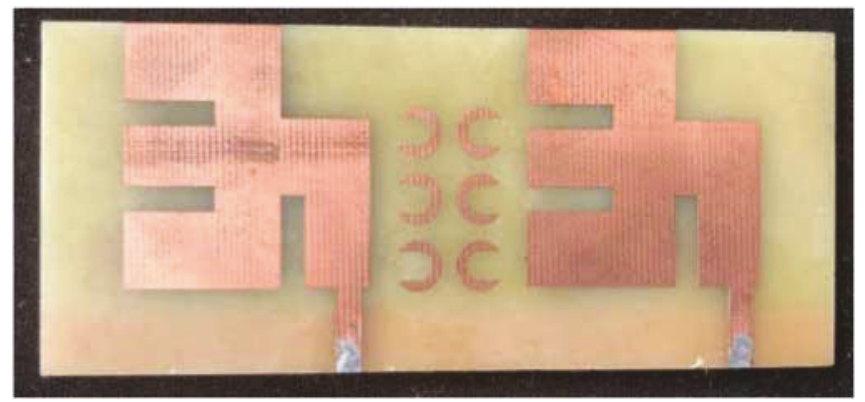

Figure 7: Fabricated antenna.

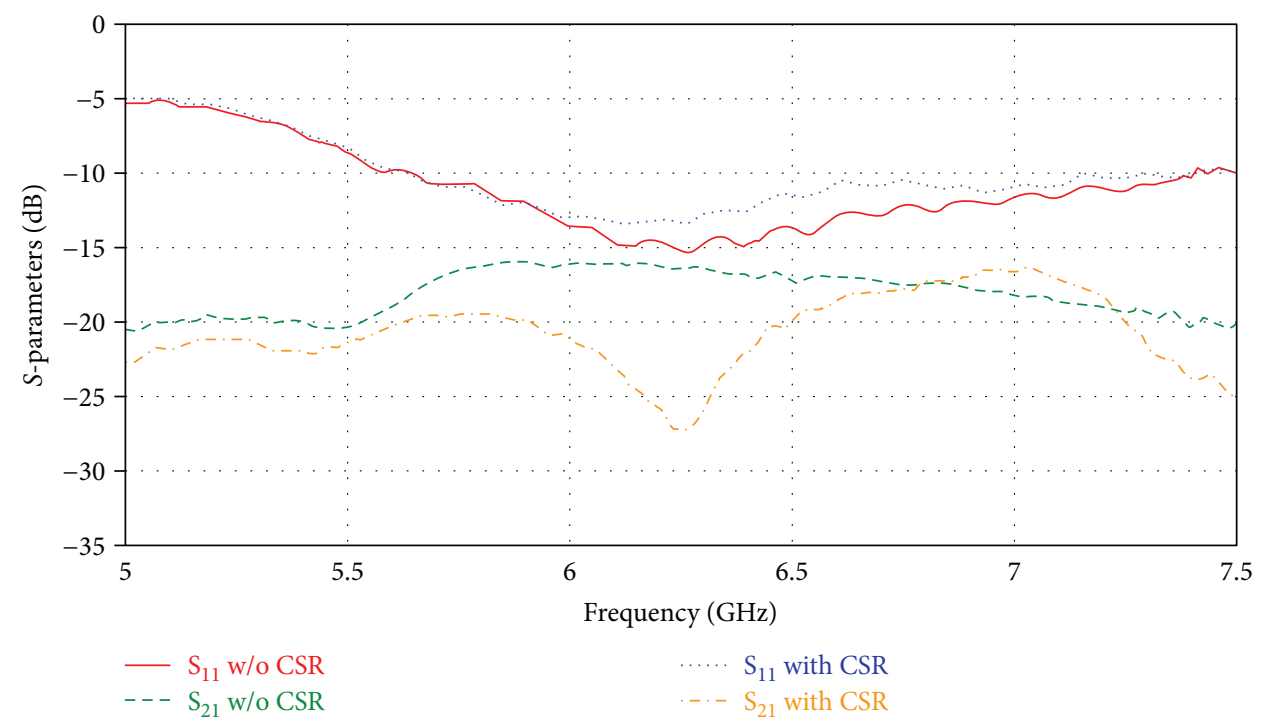

FIGURE 8: Comparison between measured S-parameters of the proposed MIMO system with and without CSR matrix structure.

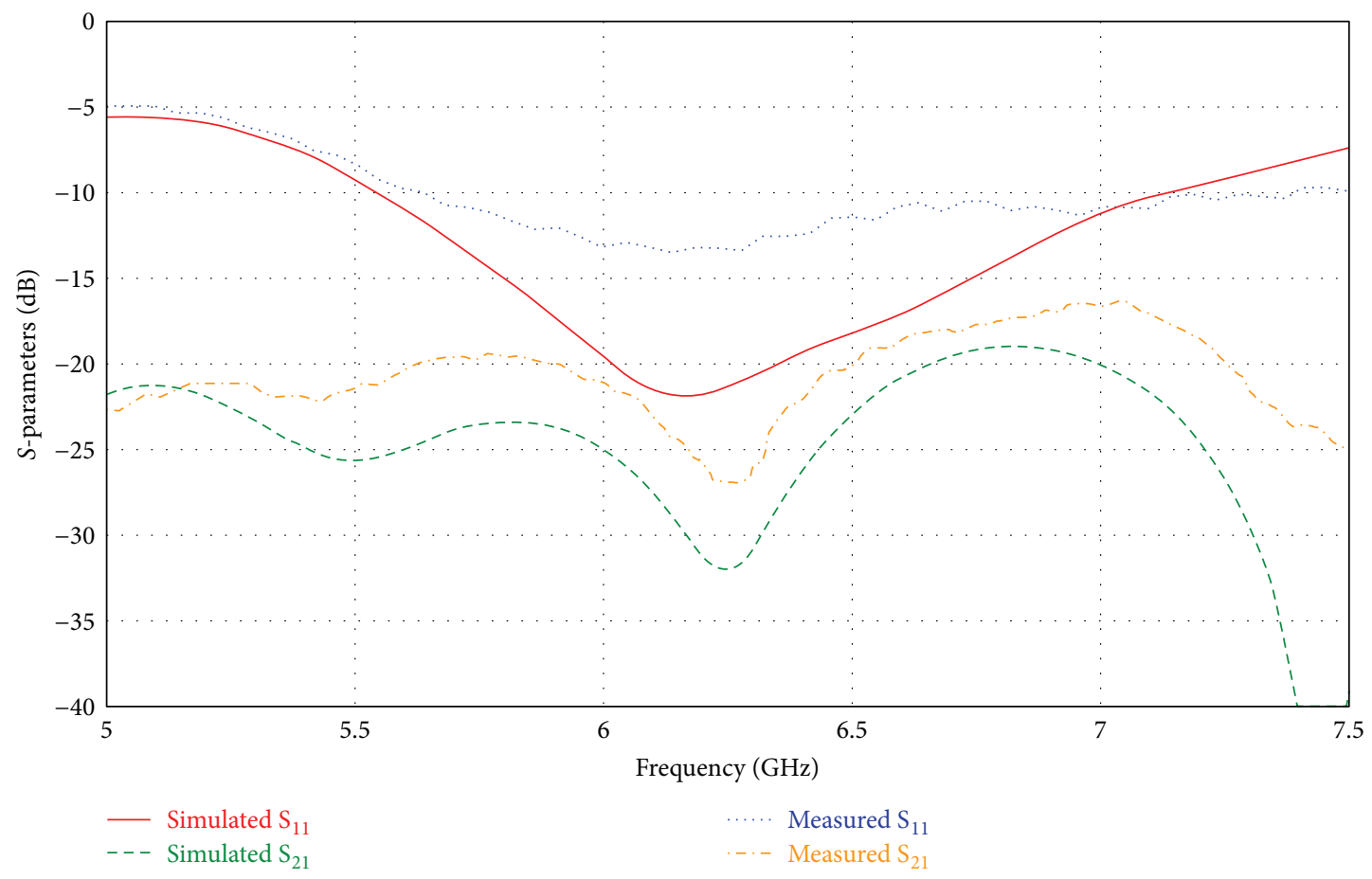

FIGURE 9: Comparison between simulated and measured S-parameters with the CSR matrix structure of the proposed MIMO system. 

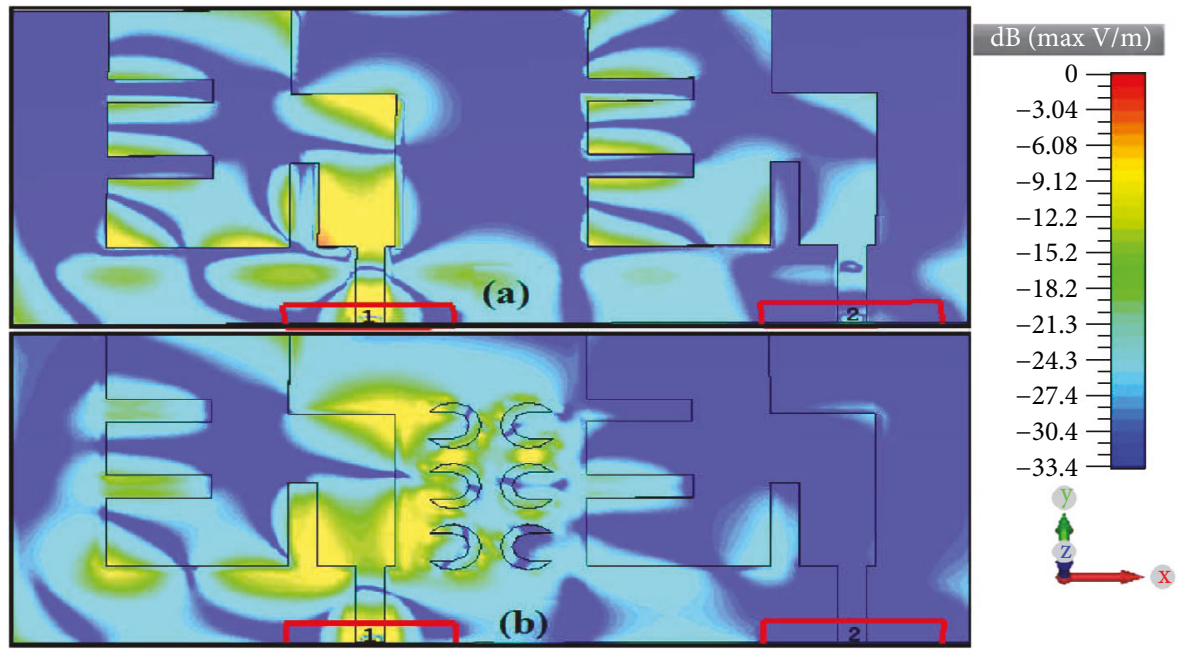

FIgURE 10: Surface current distribution (a) without CSR matrix structure (b) and with CSR matrix structure.

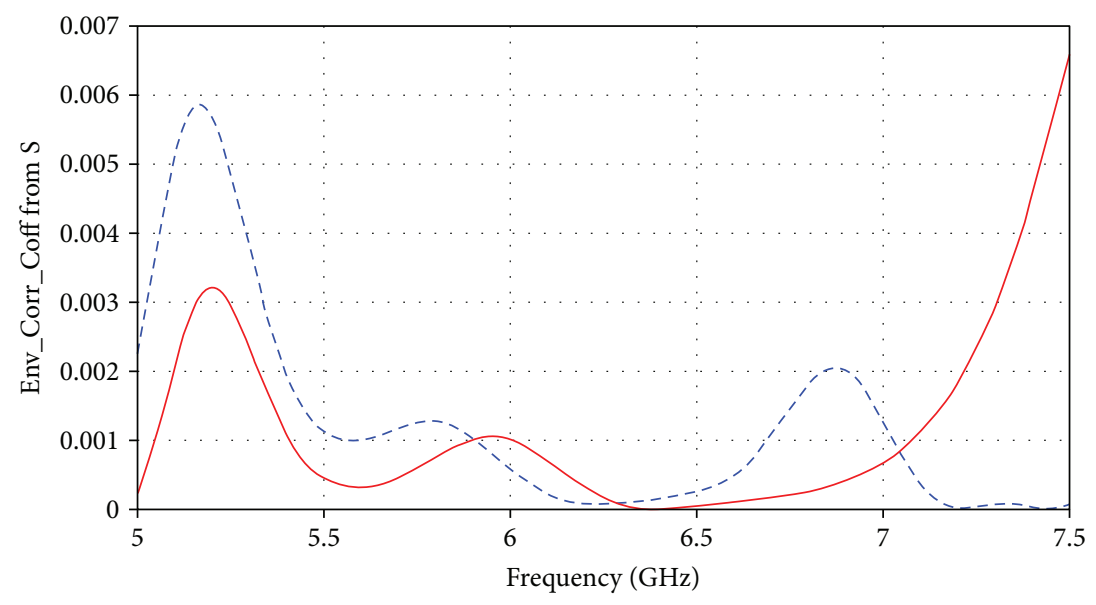

- ECC w/o CSR
--- ECC with CSR

FIGURE 11: ECC of MIMO system with and without MTM-CSR matrix structure.

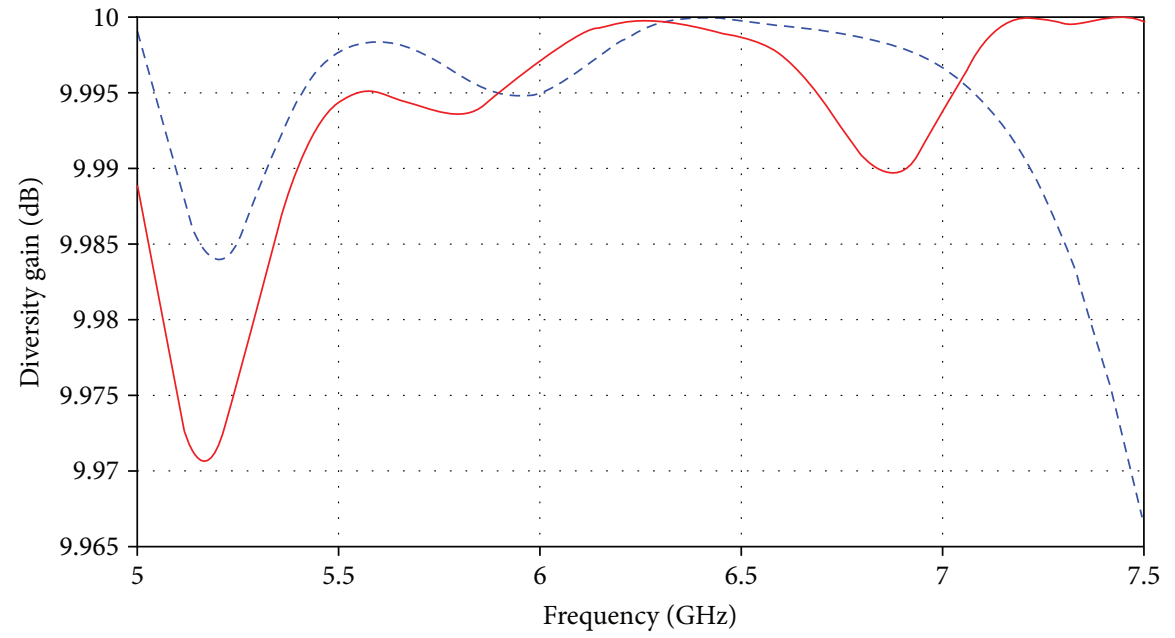

— DG w/o CSR

- - - DG with CSR

FIGURE 12: Diversity gain of the MIMO antenna with and without MTM-CSR matrix structure. 


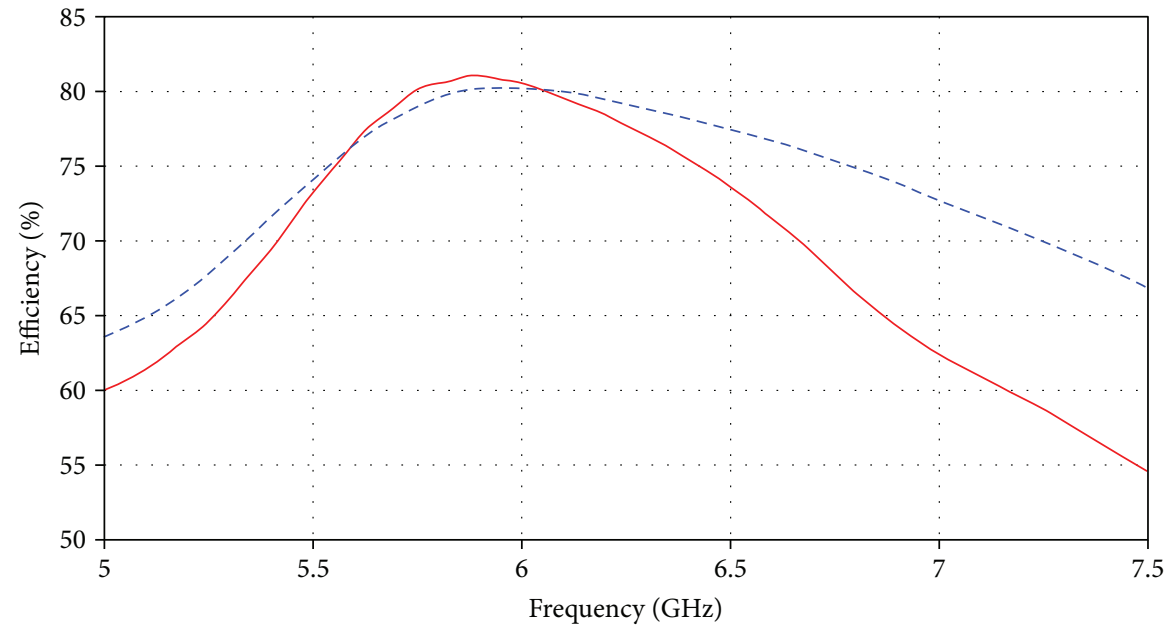

- Tot. efficiency w/o CSR

- - - Tot. efficiency with CSR

FIGURE 13: Simulated total efficiency of whole antenna system with and without MTM-CSR matrix structure.

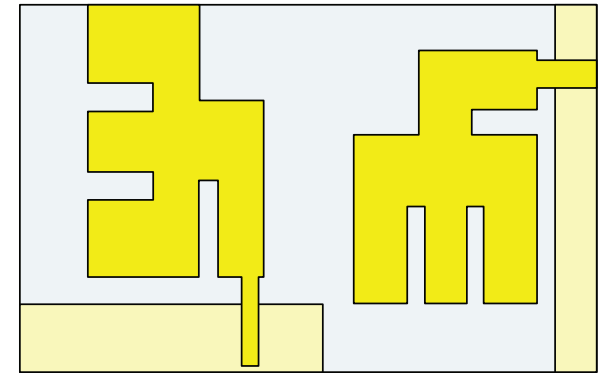

(a)

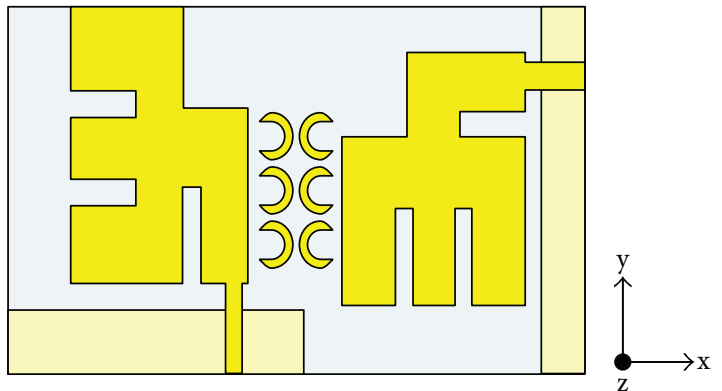

(b)

FIgURE 14: The proposed MIMO system in orthogonal configuration, (a) without CSR matrix structure, (b) and with CSR matrix structure.

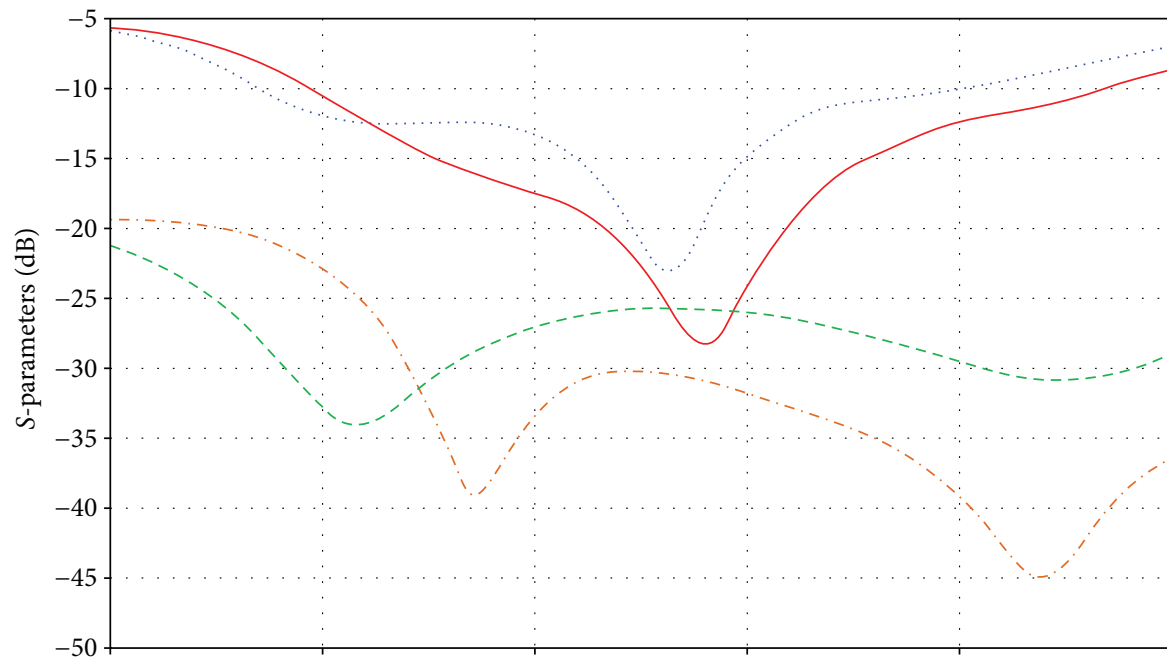

Frequency $(\mathrm{GHz})$
$-S_{11}$ w/o CSR
$\begin{array}{ll} & S_{11} \text { with CSR } \\ \cdots & S_{21} \text { with CSR }\end{array}$

FIGURE 15: Comparison between simulated S-parameters with and without CSR matrix structure. 


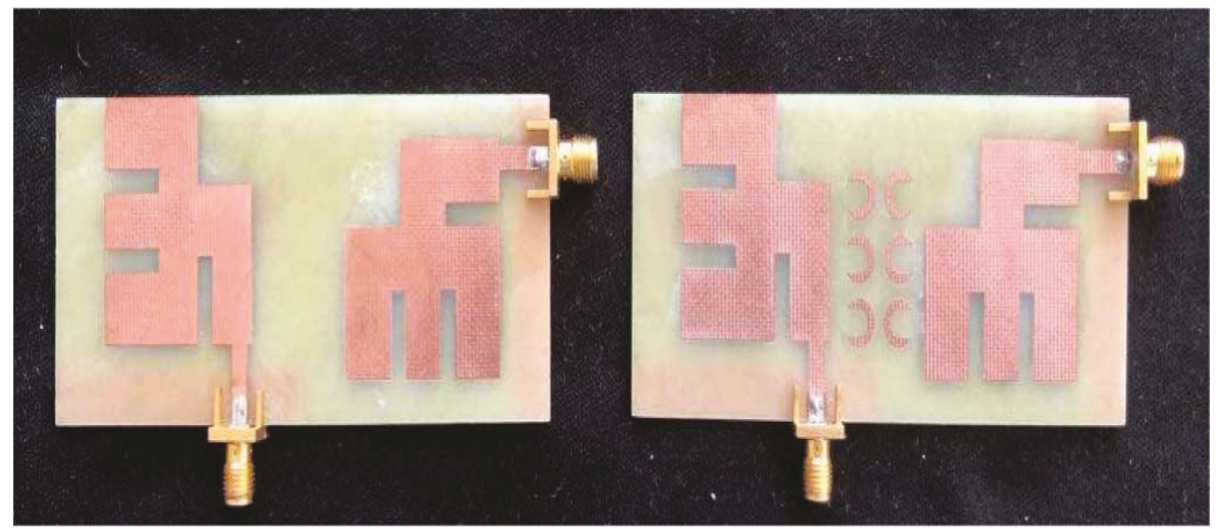

(a)

(b)

FIGURE 16: Fabricated antenna in orthogonal configuration (a) without and (b) with CSR structure.

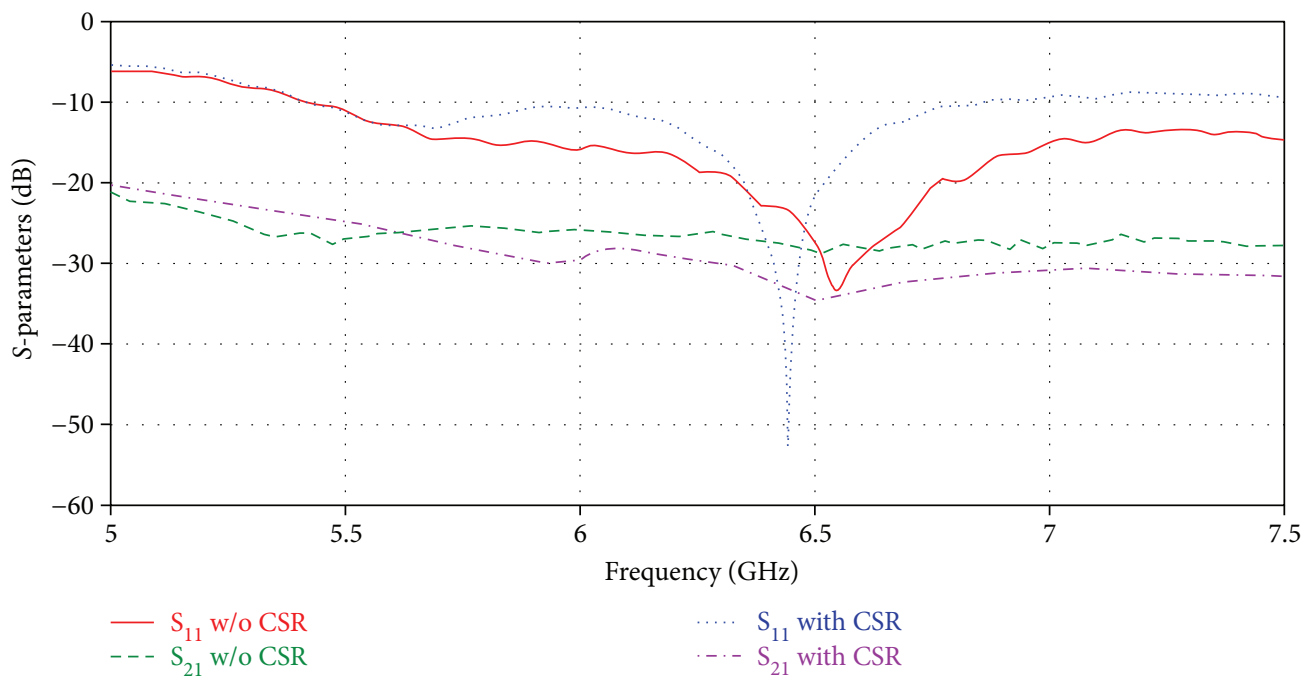

FIgURE 17: Comparison of the measured S-parameters of the modified MIMO system with and without CSR structure.

they have orthogonal polarizations as given in Figure 14(a). The same $2 \times 3$ matrix of MTM-CSR is placed between the antennas as shown in Figure 14(b). However, these antennas have been redesigned to adapt the changes in substrate and after that optimized to reduce their dimensions as compared with those presented.

Figure 15 presents the simulation results for the $S$-parameters with and without the MTM-CSR matrix structure. The mutual coupling without CSR matrix structure is now about $26 \mathrm{~dB}$ and appears to be lower than that of the structure in Figure 1(b). As seen from this figure, the proposed technique achieves a reduction in mutual coupling up to $30 \mathrm{~dB}$, which improves the isolation between antenna elements.

Figure 16 shows the prototype MIMO system in an orthogonal configuration with and without CSR structure. Figure 17 shows the comparison between the measured $S$-parameters with and without CSR structure, and Figure 18 shows the simulated and measured $S$-parameters with CSR structure. It is seen that there is a good match between the results.

By analyzing the surface current distribution, the mechanism of CSR matrix structure can be more understandable.
The mutual coupling between the two antenna elements in a MIMO system is related to the current direction that flows on the surface of the antennas. If the current flows in the same direction on the adjacent sides of both the antennas, the mutual coupling increases. Similarly, if the currents are in the opposite direction, the induced mutual coupling is suppressed. The surface waves can clearly be observed in Figure 19(a) without CSR matrix structure. Figure 19(b) shows that the induced surface current can be suppressed on the other element, which decreases the mutual coupling when the CSR matrix structure is inserted between antenna elements.

To evaluate the orthogonal MIMO system performance in terms of correlation and reliability, the ECC and DG were calculated using (3) and (4) which mentioned previously. Figure 20 shows the ECC with and without MTMCSR matrix. It is evident that ECC lower than 0.001 in both cases is pretty good, while Figure 21 shows the DG. It is clear that the DG reaches 9.99 in both cases (with and without MTM-CSR matrix) within the frequency band. This confirms that the maximum diversity gain is obtained 


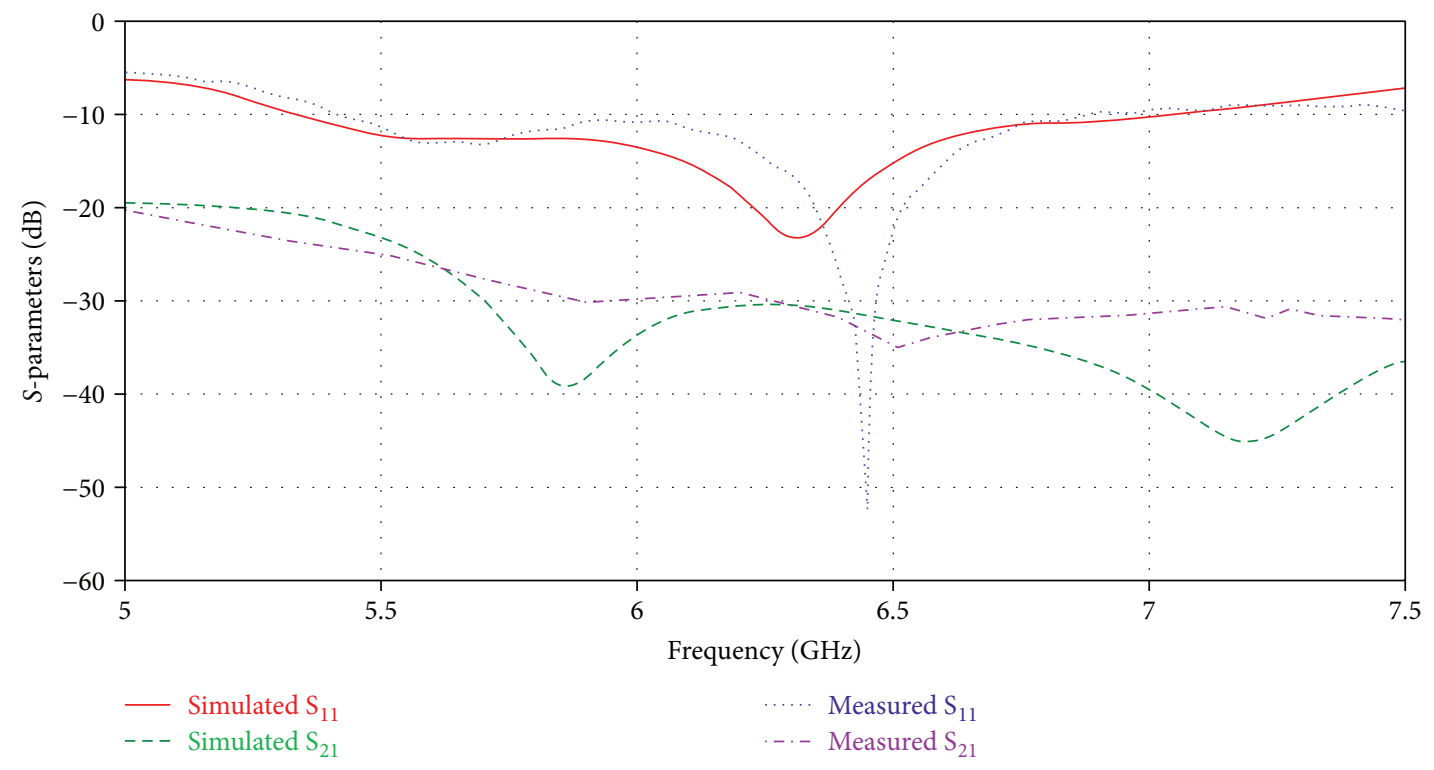

FIGURE 18: Comparison of the simulated and measured S-parameters with the CSR structure of the proposed MIMO system.

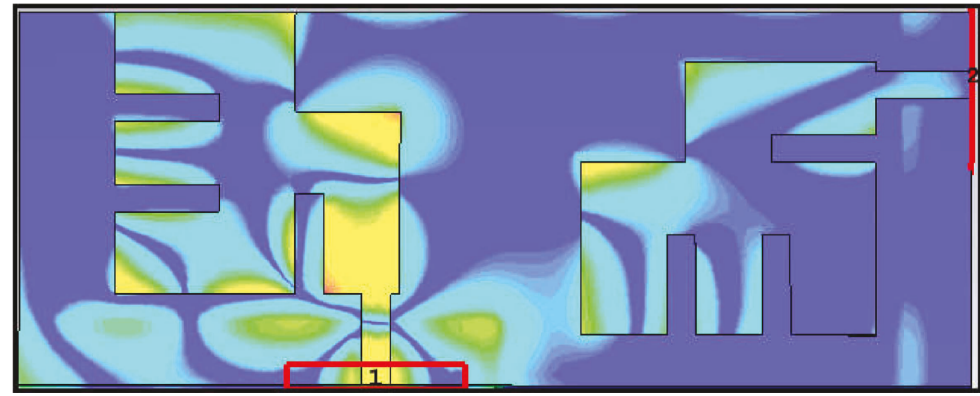

(a)

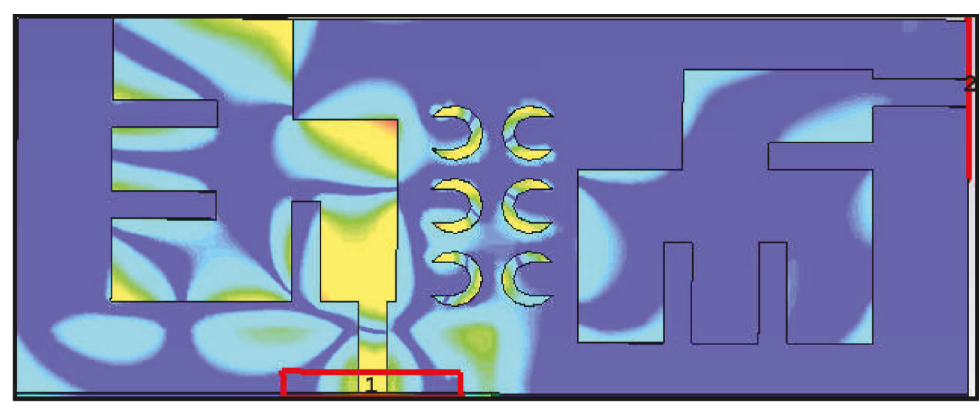

(b)
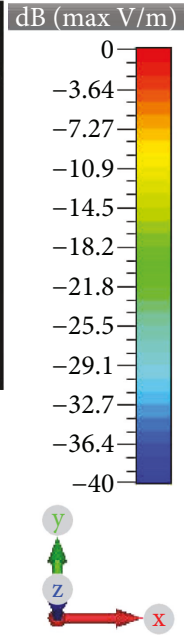

FIGURE 19: Surface current distribution (a) without and (b) with CSR matrix structure.

when the correlation coefficient is zero. Hence, the system could be classified as a high-efficiency system with good diversity performance.

Figure 22 demonstrates the simulated total efficiency of the MIMO array when the antennas are in an orthogonal configuration with and without decoupling structure MTM-CSR matrix. It is evident that the total efficiency exhibits an enhancement across the operating frequency band of the antennas in the presence of the proposed decoupling structures.

To examine the influence of the decoupling structure on the radiation pattern of the antennas, the left patch antenna was excited, and the right patch was terminated with $50 \Omega$ impedance in Figures 1, 6, 14(a), and 14(b). The proposed MIMO system is placed in $x-y$ plane. Figure 23 illustrates the simulated E-plane and H-plane of the radiation pattern of the E-shaped MIMO antenna system in a parallel configuration with and without placing CSR matrix structure between the antennas. Finally, the radiation pattern of the proposed system in an orthogonal configuration for the left and the right E-shaped MIMO antennas is shown in Figure 24. It is observed that the decoupling structure has little effect on the radiation pattern of the antenna system, which is the desired result. 


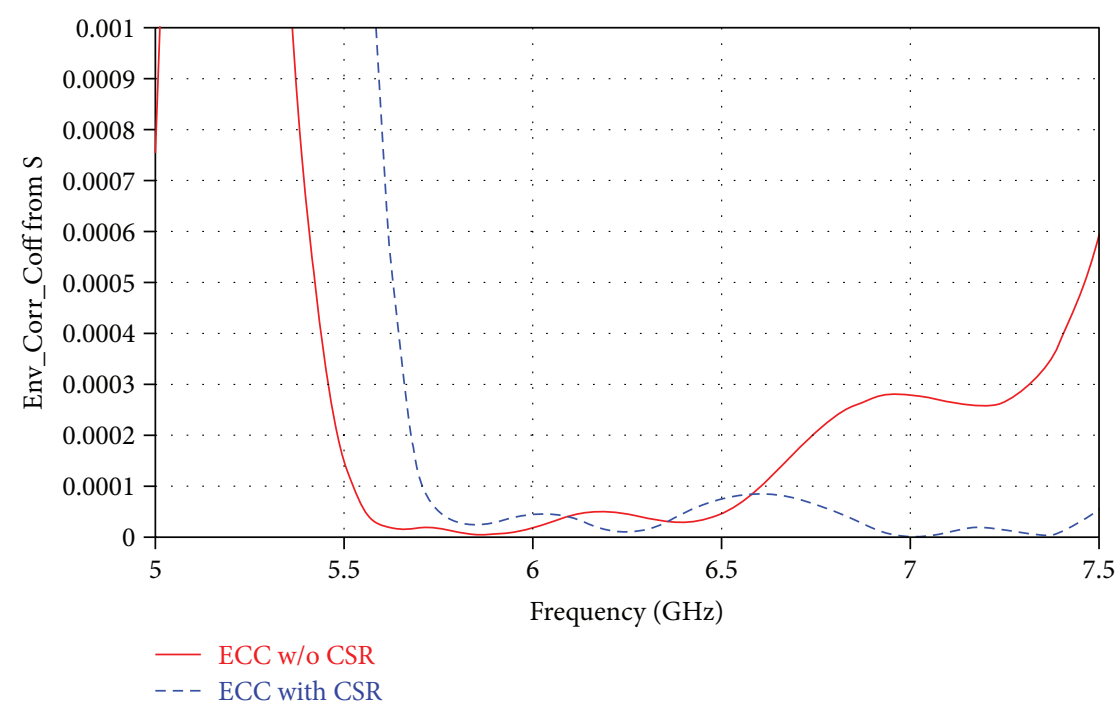

FIgURE 20: ECC of orthogonal MIMO system with and without MTM-CSR matrix structure.

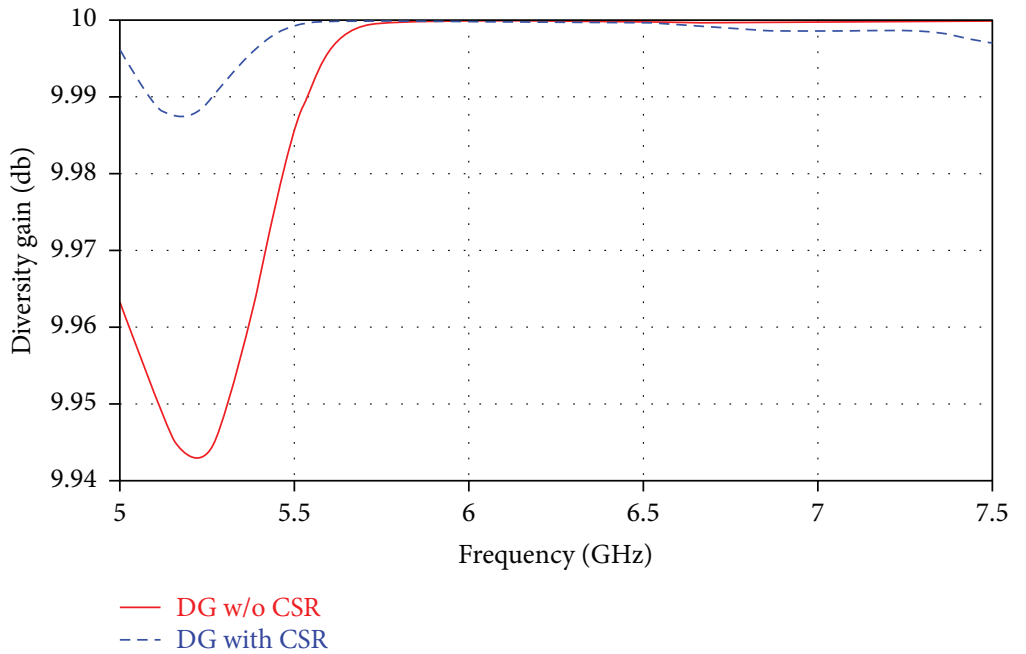

FIGURE 21: DG of the orthogonal MIMO system with and without MTM-CSR matrix structure.

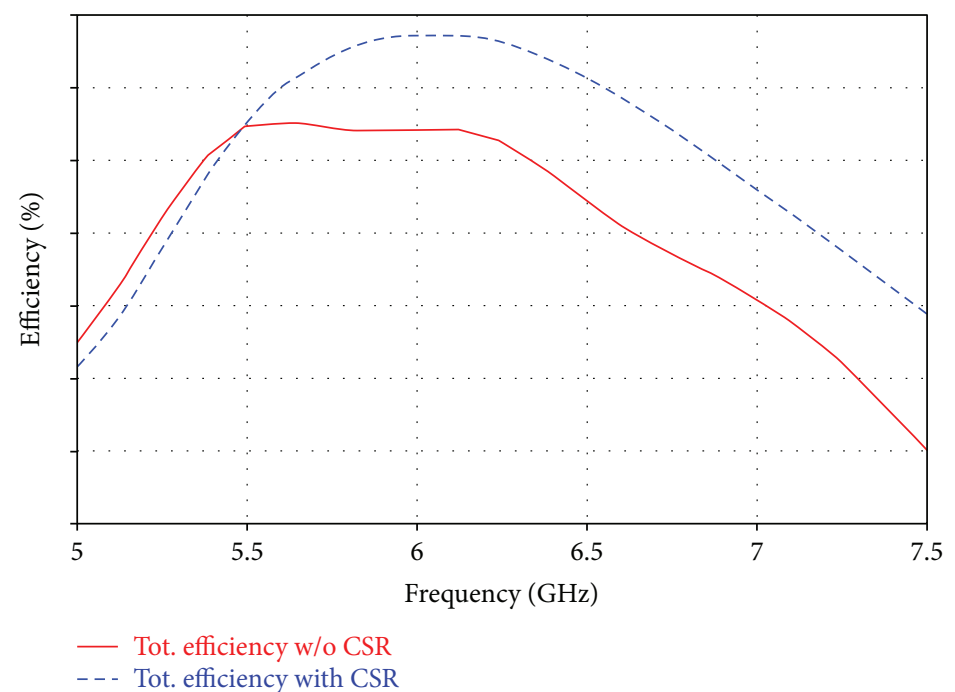

FIGURE 22: Simulated total efficiency of a whole antenna system with and without MTM-CSR matrix structure. 


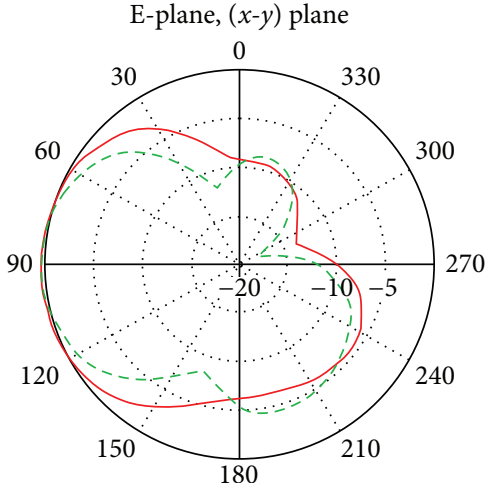

Theta/degree versus $\mathrm{dB}$

- W/O MTM-CSR

(a)

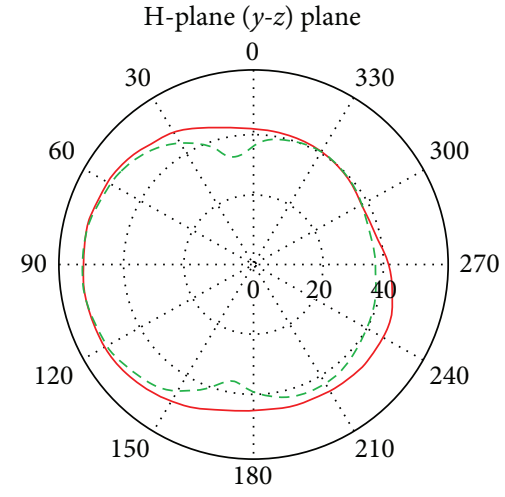

Phi/degree versus $\mathrm{dB}$

- W/o MTM-CSR

(b)

FIGURE 23: Radiation pattern of the E-shaped MIMO antenna system in parallel configuration: (a) E-plane and (b) H-plane.

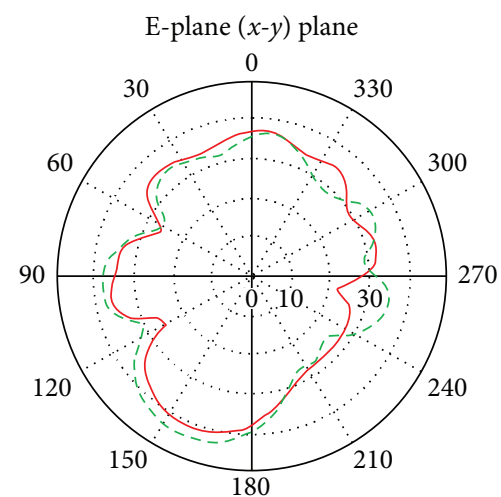

$\mathrm{Phi} /$ degree versus $\mathrm{dB}$

— W/o MTM-CSR

- - - With MTM-CSR

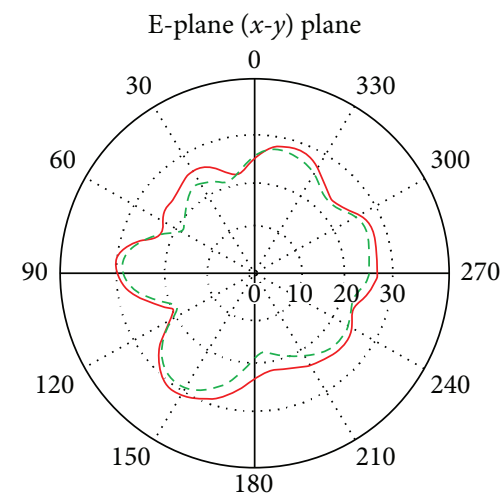

Theta/degree versus $\mathrm{dB}$

— W/o MTM-CSR

- - - With MTM-CSR

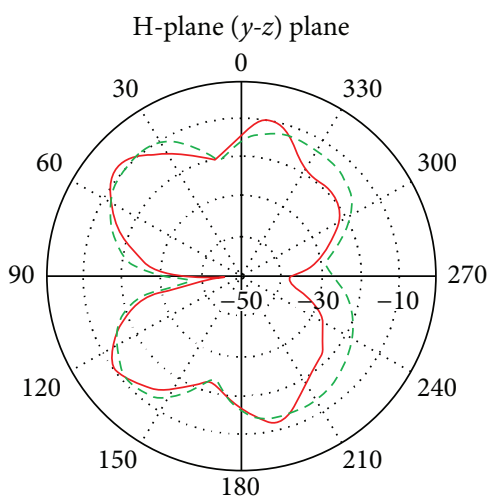

Theta/degree versus $\mathrm{dB}$

- W/o MTM-CSR

- - - With MTM-CSR

(a)

$$
\text { H-plane }(y-z) \text { plane }
$$

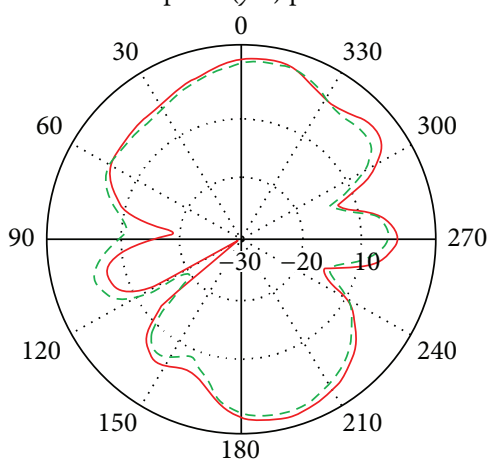

Phi/degree versus $\mathrm{dB}$

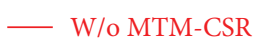

- - - With MTM-CSR

(b)

FIGURE 24: The radiation pattern of the E-shaped MIMO antenna system in the orthogonal configuration: (a) E-plane and H-plane for the left and (b) E-plane and H-plane for the right E-shaped antennas. 


\section{Conclusion}

In this work, two E-shaped antenna MIMO systems suitable for WLAN and WiMAX applications with enhanced isolation are proposed. The motive of this work is to suppress the mutual coupling between the antenna elements using metamaterial structure. This MIMO antenna system resonates at $6.3 \mathrm{GHz}$ with an impedance bandwidth of $34.42 \%$ in the range of frequencies from $5-7.5 \mathrm{GHz}$. The coupling reduction has been achieved by printing a $2 \times 3$ MTMCSR matrix between array elements. This structure can block the surface waves inside the substrate of the antenna and guide them in another direction. The mutual coupling in the E-shaped MIMO antenna system is investigated with and without the $2 \times 3$ CSR matrix in both configurations, parallel and orthogonal antenna arrangement. The isolation of MIMO antennas with $2 \times 3$ MTM-CSR decoupling structure is shown to improve about $12 \mathrm{~dB}$, whereas it is improved in orthogonal arrangement around $6 \mathrm{~dB}$ and maintaining the overall mutual coupling less than $-20 \mathrm{~dB}$ in the operating band.

\section{Conflicts of Interest}

The authors declare that they have no conflicts of interest.

\section{References}

[1] W. C. Y. Lee, Mobile Communications Engineering, Wiley, New York, NY, USA, 1982.

[2] R. G. Vaughan and J. B. Andersen, "Antenna diversity in mobile communications," IEEE Transactions on Vehicular Technology, vol. 36, no. 4, pp. 149-172, 1987.

[3] J. S. Colburn, Y. Rahmat-Samii, M. A. Jensen, and G. J. Pottie, "Evaluation of personal communications dual-antenna handset diversity performance," IEEE Transactions on Vehicular Technology, vol. 47, no. 3, pp. 737-746, 1998.

[4] A. Diallo, C. Luxey, P. Le Thuc, R. Staraj, and G. Kossiavas, "Enhanced two-antenna structures for universal mobile telecommunications system diversity terminals," IET Microwaves, Antennas \& Propagation, vol. 2, no. 1, pp. 93-101, 2008.

[5] C.-H. Lee, S.-Y. Chen, and P. Hsu, "Integrated dual planar inverted-F antenna with enhanced isolation," IEEE Antennas and Wireless Propagation Letters, vol. 8, pp. 963-965, 2009.

[6] F. Yang and Y. Rahmat-Samii, "Microstrip antennas integrated with electromagnetic band-gap (EBG) structures: a low mutual coupling design for array applications," IEEE Transactions on Antennas and Propagation, vol. 51, no. 10, pp. 2936-2946, 2003.

[7] Z. Iluz, R. Shavit, and R. Bauer, "Microstrip antenna phased array with electromagnetic bandgap substrate," IEEE Transactions on Antennas and Propagation, vol. 52, no. 6, pp. 14461453, 2004.

[8] F. Caminita, S. Costanzo, G. Di Massa et al., "Reduction of patch antenna coupling by using a compact EBG formed by shorted strips with interlocked branch-stubs," IEEE Antennas and Wireless Propagation Letters, vol. 8, pp. 811-814, 2009.

[9] Y. Yu, L. Yi, X. Liu, and Z. Gu, "Mutual coupling reduction of dual-frequency patch antenna arrays," ACES Journal, vol. 31, no. 9, pp. 1092-1099, 2016.
[10] L. H. Weng, Y. C. Guo, X. W. Shi, and X. Q. Chen, "An overview on defected ground structure," Progress In Electromagnetics Research B, vol. 7, pp. 173-189, 2008.

[11] E. J. Rothwell, J. L. Frasch, and S. M. Ellison, "Analysis of the Nicolson-Ross-Weir method for characterizing the electromagnetic properties of engineered materials," Progress In Electromagnetics Research, vol. 157, pp. 31-47, 2016.

[12] A. Habashi, J. Nourinia, and C. Ghobadi, "Mutual coupling reduction between very closely spaced patch antennas using low-profile folded split-ring resonators (FSRRs)," IEEE Antennas and Wireless Propagation Letters, vol. 10, pp. 862-865, 2011.

[13] M. M. Bait-Suwailam, O. F. Siddiqui, and O. M. Ramahi, "Mutual coupling reduction between microstrip patch antennas using slotted-complementary split-ring resonators," IEEE Antennas and Wireless Propagation Letters, vol. 9, pp. 876878, 2010.

[14] M. S. Sharawi, "Printed multi-band MIMO antenna systems and their performance metrics [wireless corner]," IEEE Antennas and Propagation Magazine, vol. 55, no. 5, pp. 218-232, 2013. 


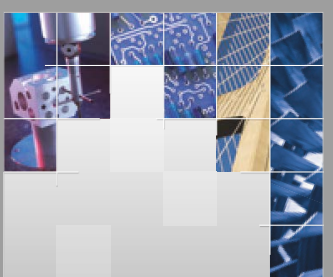

\section{Enfincering}
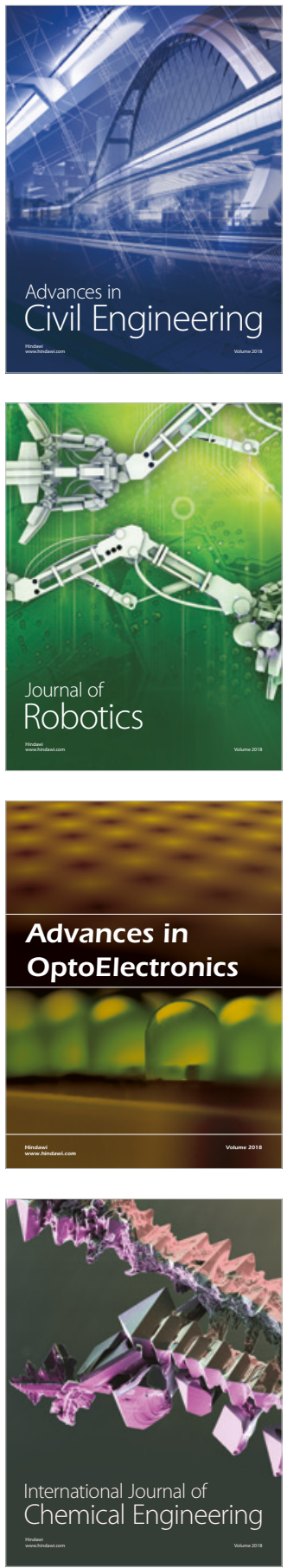

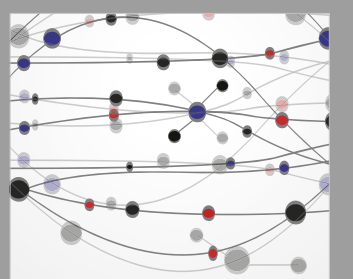

\section{Rotating \\ Machinery}

The Scientific World Journal

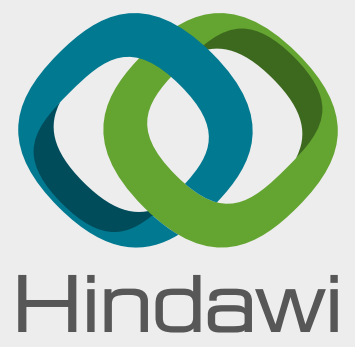

Submit your manuscripts at

www.hindawi.com
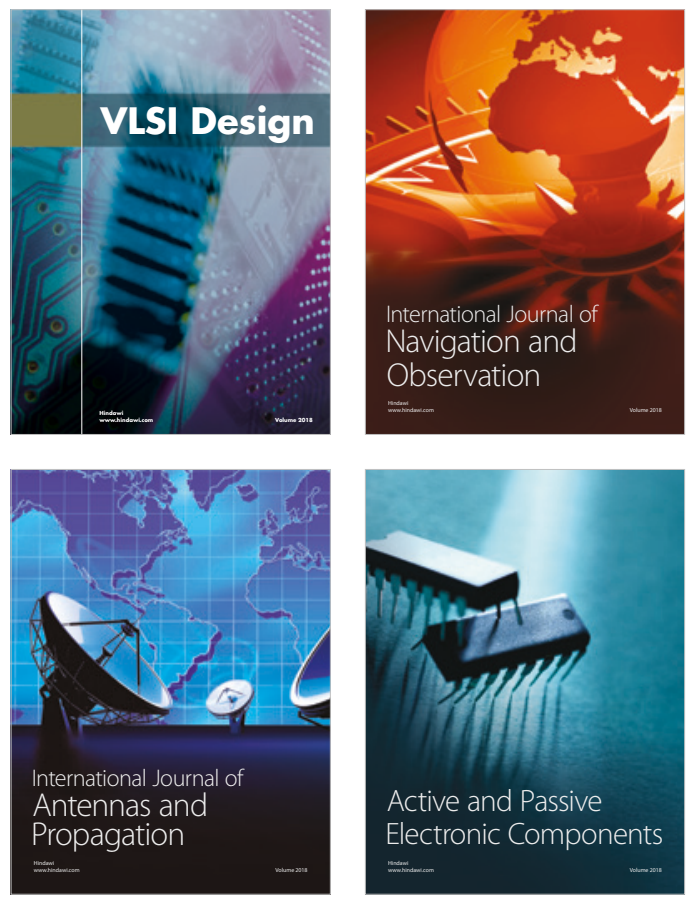
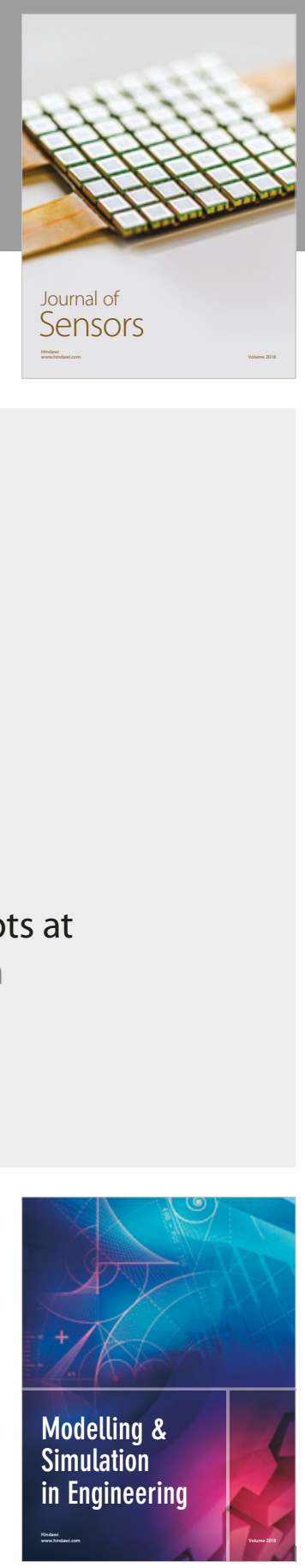

\section{Advances \\ Multimedia}
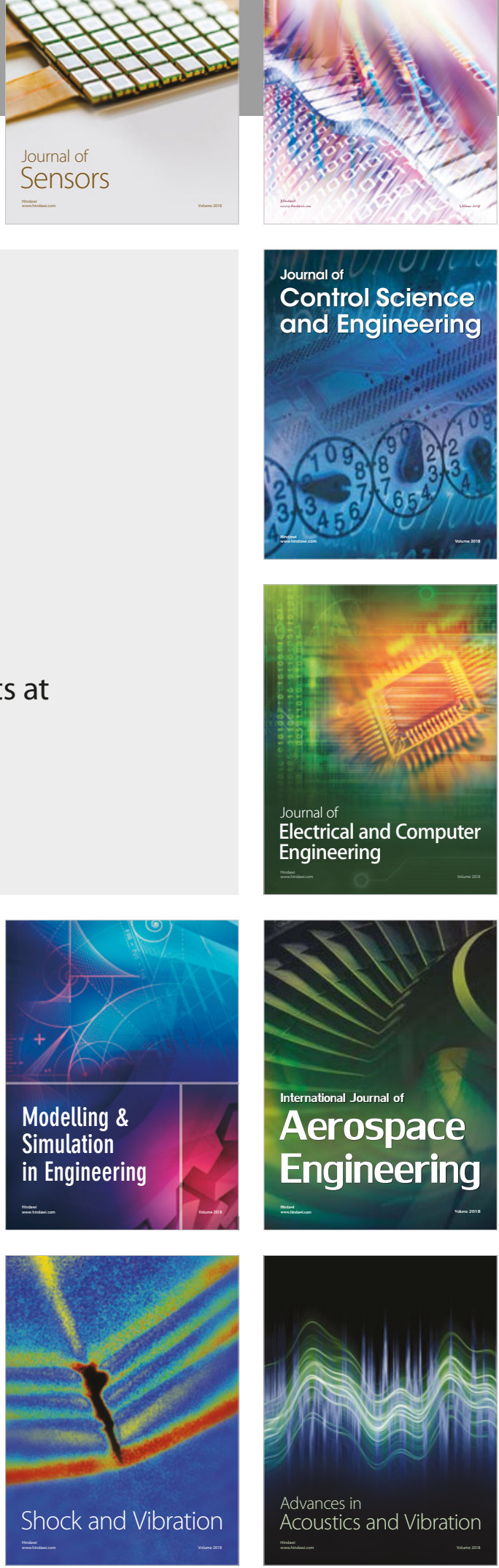